MATEC Web of Conferences 26, 01006

(2015)

DOI: $10.1051 /$ matecconf/ 20152601006

(C) Owned by the authors, published by EDP Sciences, 2015

\title{
CdSe Nanoparticles with Clean Surfaces: Gas Phase Synthesis and Optical Properties
}

\author{
Hongwei Zhang ${ }^{1, a}$, Xing Peng ${ }^{1, b}$, Lin Sun ${ }^{1, c}$, Fei Liu ${ }^{2 . d}$ \\ ${ }^{1}$ Department of Materials Science and Engineering, Nanjing University, Nanjing 210093, China \\ ${ }^{2}$ Suzhou SinoRaybo Nanoscience and Nanotech Co., Ltd, Suzhou Industrial Park, 215123, Suzhou, Jiangsu, China
}

\begin{abstract}
CdSe nanoparticles (NPs) were generated in gas phase with a magnetron plasma gas aggregation cluster beam source. Coagulation-free CdSe nanocrystals with very clean particle surface and interface, as well as a fairly uniform spatial distribution were obtained. The deposited NPs have a good dispersity with a mean diameter of about $4.8 \mathrm{~nm}$. A strong photoluminescence band corresponding to the near- band-edge transition of the CdSe NPs was observed. The CdSe NP films show a significant photoconductance induced by laser irradiation. With an applied bias voltage of $10 \mathrm{~V}$, the photo- induced current can be as high as $0.4 \mathrm{~mA}$ under $0.01 \mathrm{~mW} / \mathrm{mm}^{2} 405 \mathrm{~nm}$ laser illumination. Our approach offers an alternative method for CdSe NP synthesis, which has the advantages such as high purity, good process and product control, as well as mass production, as compared to the existing methods.
\end{abstract}

\section{Introduction}

Semiconductor nanoparticles (NPs) have attracted much attentions in the past two decades both in the areas of fundamental research and technical applications, such as light-emitting devices, biological label, biosensor, solar cells, as well as quantum-dot lasers [1-7]. CdSe NPs are arguably the best studied of all semiconductor nanomaterials. The recent interest in utilizing CdSe NPs for harvesting light energy has drawn great attention [8, 9]. CdSe have relatively small bandgaps and thus are capable of harvesting photons in the visible and infrared region. Various approaches have been explored to realize controllable synthesis of monodisperse CdSe NPs in the past decade. CdSe NPs in solution have been made following synthetic chemical methods. The conventional synthesis of CdSe NPs uses large volumes of high-boiling organic solvents at high temperatures into which aggressive and toxic chemicals must be injected quickly and reproducibly. Large-scale production of CdSe NPs is particularly difficult because temperature control and homogeneous mixing are difficult for fast reactions in large volumes, but critical for particle size uniformity. To overcome the limitations of the above methodologies, laser ablation in liquid environments [10] and continuous chemical aerosol flow synthesis [11] have been used recently.

Control of size and surface structure continues to be of interest in the semiconductor NPs investigation. CdSe NPs were usually capped with various organic species, such as surfactants, which give rise to a barrier to aggregation and electronic passivation of the NPs. However, for some application, such as photoconducting devices, dense arrays of NPs with clean particle surface and interface are required.

Synthesis of NPs with gas-phase process may have some advantages in some cases because they enable high purity, good process and product control, as well as mass production. In this paper, we fabricate dense arrays of CdSe NPs by gas phase cluster beam deposition and investigate their photoluminescence and photoconducting properties. Our approach offers an alternative method for CdSe NP synthesis as compared to the existing methods.

\section{Experimental}

We used high vacuum cluster beam deposition system to prepare CdSe NPs. The gas-phase nanoclusters to be deposited were generated in a magnetron plasma gas aggregation cluster source described elsewhere in detail $[12,13]$. The formation of CdSe NPs in gas phase is based on homogeneous nucleation with the present of inert buffer gas and subsequent condensation and growth. Gas-phase molecules were sputtered from CdSe solid target through magnetron discharge to create a high degree of supersaturation, thereby inducing a high nucleation density. The magnetron discharge was operated in a liquid nitrogen cooled aggregation tube. A stream of high purity (99.99\%) argon gas was introduced closely to the CdSe target surface. The flow rate was fixed at $90 \mathrm{sccm}$. Another stream of helium gas was fed as a buffer gas near the magnetron discharge head. The buffer gas flow rate was fixed at $50 \mathrm{sccm}$. A stable pressure of $100 \mathrm{~Pa}$ was maintained in the aggregation tube through the differential pumping system. CdSe NPs

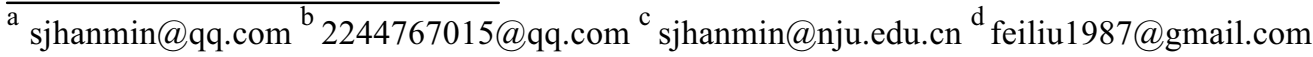


were formed and immediately swept by the buffer gas stream out of aggregation tube into high vacuum through a nozzle to quench the NP growth. The NPs were then deposited on fused silica substrates in high vacuum at room temperature. The deposition rate was monitored by a quartz crystal microbalance and maintained at $2 \AA /$ s. For photoconductance measurement, CdSe NP layers with controlled coverage were deposited on pre-deposited gold interdigital electrodes. The electrodes were $100 \mathrm{~nm}$ in thickness with $5 \mu \mathrm{m}$ electrode separation.

The size and morphology of the CdSe NPs were characterized with a transmission electron microscope (TEM, FEI TECNAI F20s TWIN). For TEM characterization, amorphous carbon films supported with copper grids were used for deposition. The composition of the CdSe NPs was determined by using X-ray photoelectron spectroscopy (XPS). The Al Ka x-ray source $(1486.6 \mathrm{eV})$ was used for excitation. The $\mathrm{C} 1 \mathrm{~s}$ line of $284.8 \mathrm{eV}$ binding energy was used as a reference to correct the binding energies for the charge shift. The composition of the CdSe NPs was also characterized by Raman scattering spectroscopy (NT-MDT NTEGRA Spectra, with $473 \mathrm{~nm}$ laser excitation). Spectrophotometric and photoluminescence (PL) spectra measurements were performed on a spectrograph equipped with a Zolix omni-180i monochromator, a photomultiplier. For photoconductance measurement, the sample was illuminated with a $405 \mathrm{~nm}$ diode laser. The conductance of the sample was measured by measuring the current in the sample with a source meter (Keithley 2400) for a bias voltage applied to the interdigital electrodes.
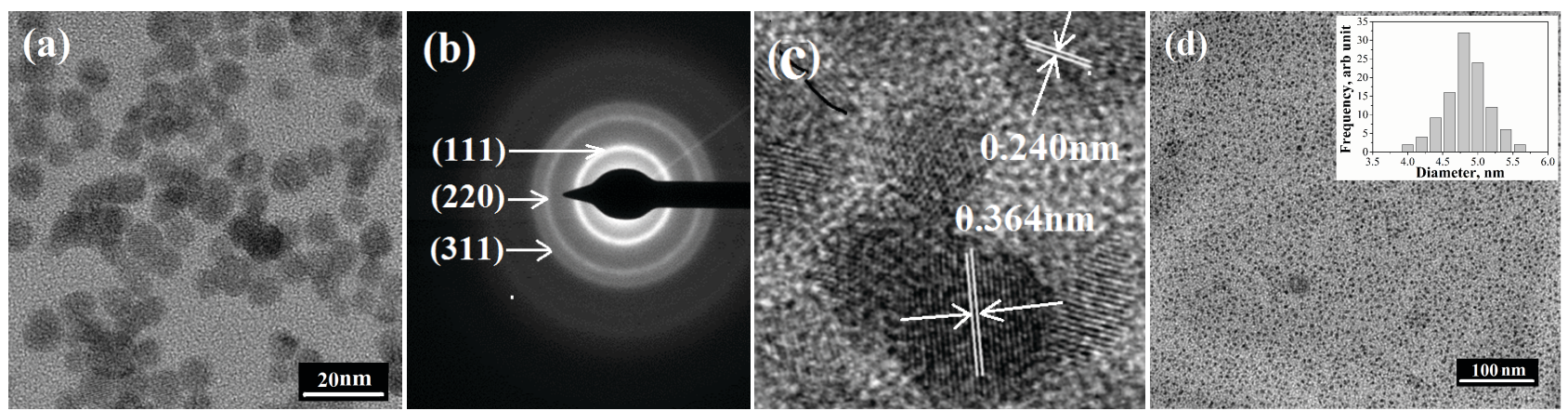

Figure. 1. (a)A TEM image of CdSe nanoparticles deposited on amorphous carbon film surface. (b) Selected area electron diffraction pattern of the CdSe nanoparticles. (c) A HRTEM image of the CdSe nanonanoparticles. (d) a large area TEM image of the CdSe nanoparticles with a plot of their size distribution shown as inset.

\section{Results and discussions}

TEM images of typical low coverage deposited samples on amorphous carbon films of TEM grid are shown in Fig. 1. NPs are found to be evenly distributed on the TEM grid and their sizes are apparently uniform with nearly spherical shape. Individual NPs with size of several nanometers can be clearly distinguished. No obvious coagulation among the particles could be observed. The diameters of the smallest ones are around $3.5 \mathrm{~nm}$, which may represent the size of the original clusters from the gas aggregation process. The mean diameter of the NPs was measured to be around $4.8 \mathrm{~nm}$. Selected area electron

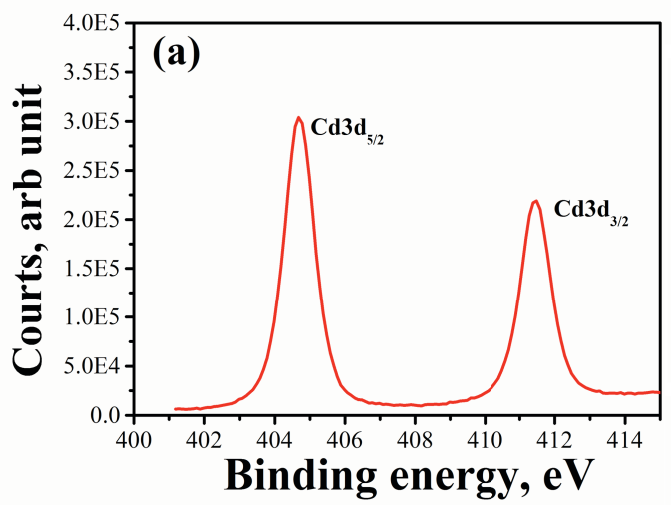

diffraction (Fig. 1b) of the NP arrays indicates that the NPs are well crystallized with a wurtzite structure as bulk CdSe. Figure 1c illustrates the HRTEM images of the CdSe NPs, in which well-resolved lattice fringes could be found. We indicate in the figure two lattice spacing of the NPs, which are measured equal to $0.240 \mathrm{~nm}$ and $0.354 \mathrm{~nm}$ respectively, and corresponds to the lattice spacing between $\{111\}$ planes and $\{220\}$ planes for hexagonal CdSe nanocrystals. In Figure 1d, a large area TEM image of the CdSe NP film is shown. We can see the NPs show a fairly uniform distribution on the long range scale, having the character of a statistical deposition from the gas phase.

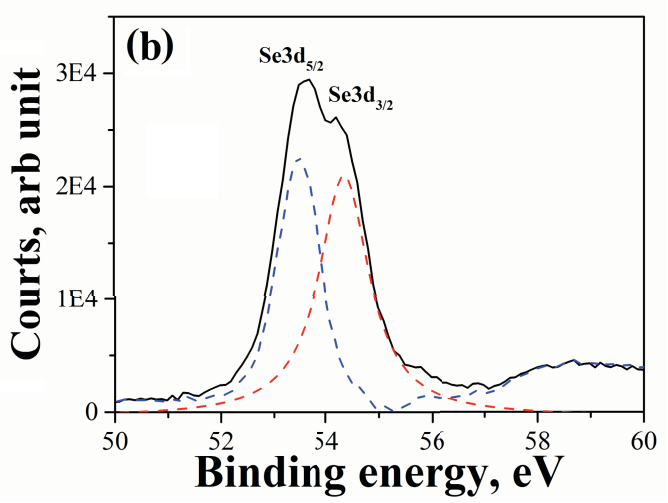


Figure. 2. (a) Cd 3d core level spectra of CdSe nanoparticles; (b) Se 3d core level spectra of CdSe nanoparticles.

Fig. 2 shows the photoemission data of $\mathrm{Cd} 3 \mathrm{~d}$ core level as well as Se $3 \mathrm{~d}$ core level measured from the NP films deposited on the silica substrates. The binding energy of $\mathrm{Cd} 3 \mathrm{~d}_{5 / 2}$ and $\mathrm{Cd} 3 \mathrm{~d}_{3 / 2}$ core level were observed at about $404.7 \mathrm{eV}$ and $411.5 \mathrm{eV}$, which agrees well with the standard data for bulk CdSe. The Se 3d photoemission peak can be deconvoluted into two components. A peak fit resolved spin-orbit splitting $3 \mathrm{~d}_{3 / 2}$ and $3 \mathrm{~d}_{5 / 2}$ peaks at $53.5 \mathrm{eV}$ and $54.3 \mathrm{eV}$ respectively, which are signatures of $\mathrm{Se}$ in $\mathrm{CdSe}$ compound. The photoemission data of Fig. 2 were not changed after the sample surface was cleaned with Ar ion sputtering, indicating that the nanoparticles were completely pure CdSe.

Fig. 3 shows a typical Raman spectrum of the CdSe NP film measured at room temperature with $473 \mathrm{~nm}$ laser excitation. The spectrum exhibits intense peaks at 205 $\mathrm{cm}^{-1}$ and $412 \mathrm{~cm}^{-1}$ respectively. The peaks can be assigned to the longitudinal optical (LO) phonon mode and its overtone (2LO). As we known, the LO phonon mode for bulk CdSe is at $210 \mathrm{~cm}^{-1}$ [14]. The downward shift of the LO phonon mode of CdSe NPs can be attributed to the quantum confinement effect of phonons in NPs, which were commonly observed for CdSe NPs smaller than $10 \mathrm{~nm}[15,16]$.

Fig. 4. shows Tauc-Plot of the optical extinction spectra of CdSe NPs. From Tauc-Plot, the band gap energy of the CdSe NPs can be determined as $2.2 \mathrm{eV}$, which is much greater than the $1.7 \mathrm{eV}$ band-gap of bulk $\mathrm{CdSe}$, indicating that quantum confinement occurs significantly in the NPs.

PL spectra were measured from $20 \mathrm{~K}$ to $280 \mathrm{~K}$ under $405 \mathrm{~nm}$ laser excitation. The CdSe NPs show a strong PL peak around 590nm $(2.1 \mathrm{eV})$, as shown in Fig. 5. The PL band is consistent well with the absorption edge observed from the extinction spectra shown in Fig. 4, indicating that the PL emission can be attributed to the fundamental transition between the energy bands. The PL peak intensity increases with the decreasing of temperature from $280 \mathrm{~K}$ to $20 \mathrm{~K}$. It also shows a red-shift from $40 \mathrm{~K}$ to $280 \mathrm{~K}$, due to the temperature-dependent band-gapshrinkage. In the low temperature region between $20 \mathrm{~K}$ and $40 \mathrm{~K}$, the PL band shows a blue shift with the temperature, which can be attributed to the size distribution of the NPs [17]. No significant PL band from defect energy levels was observed.

The photoconductance of the CdSe NP arrays was also measured with a $405 \mathrm{~nm}$ laser illumination. The current-voltage (I-V) curves of the CdSe NP arrays measured with and without light illumination are shown in Fig. 6. In the absence of irradiation, the I-V characteristic of the NP arrays has a sigmoid shape and is distinctly nonlinear, demonstrating that the NP arrays are strongly non-ohmic. With an applied bias voltage of $1 \mathrm{~V}$, the current transport in the film was measured to be about 1nA. The basic electron transport mechanism keeps nonchange under light illumination, however, the conductance of the NP arrays increases significantly. As shown in Fig. 6, illuminated with $405 \mathrm{~nm}$ laser, the current was significantly enhanced. Nearly one order of magnitude enhancement on the conductance of the NP arrays can be observed. With an applied bias voltage of $10 \mathrm{~V}$, the photo-induced current can be as high as $0.4 \mu \mathrm{A}$ under $0.01 \mathrm{~mW} / \mathrm{mm}^{2}$ laser irradiation.

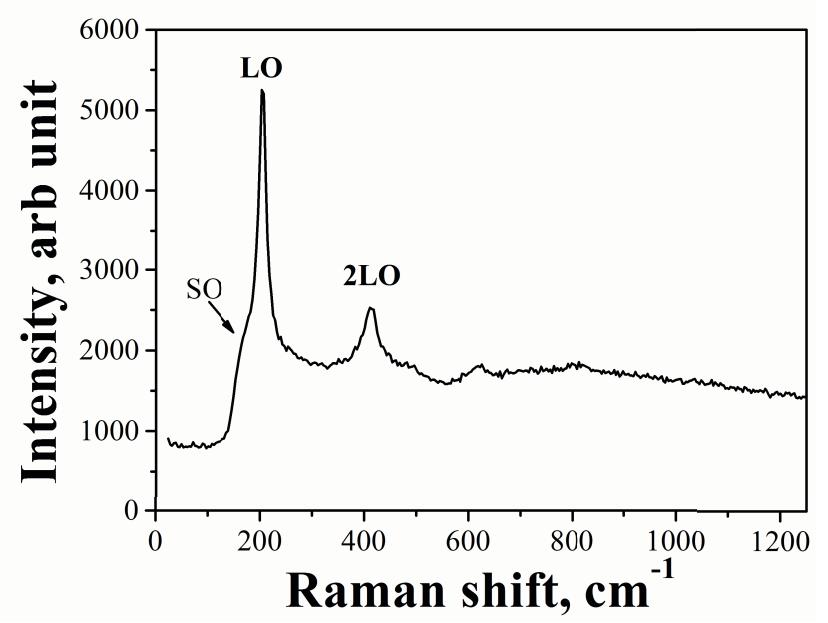

Figure. 3. Raman spectrum of the CdSe nanoparticle film.

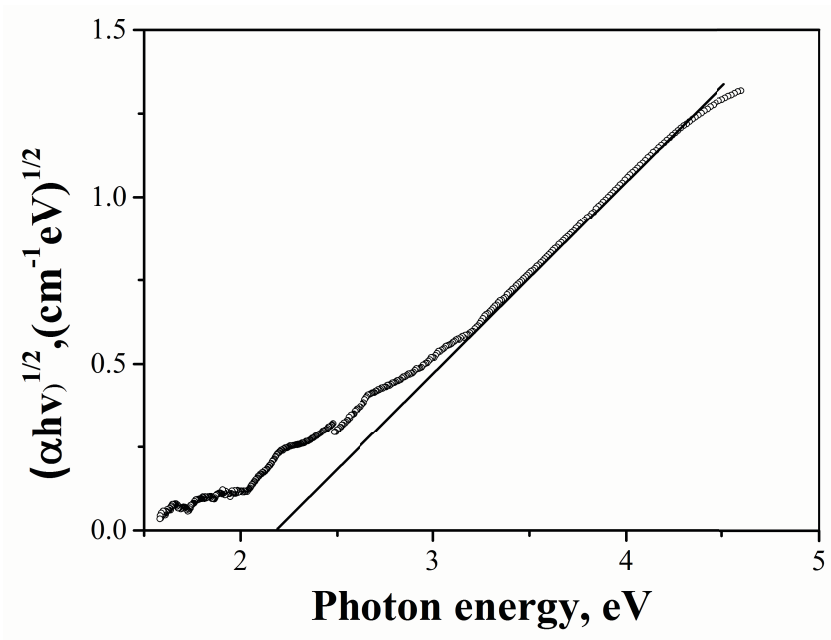

Figure. 4. Tauc-Plot of the optical extinction spectra of CdSe nanoparticles.

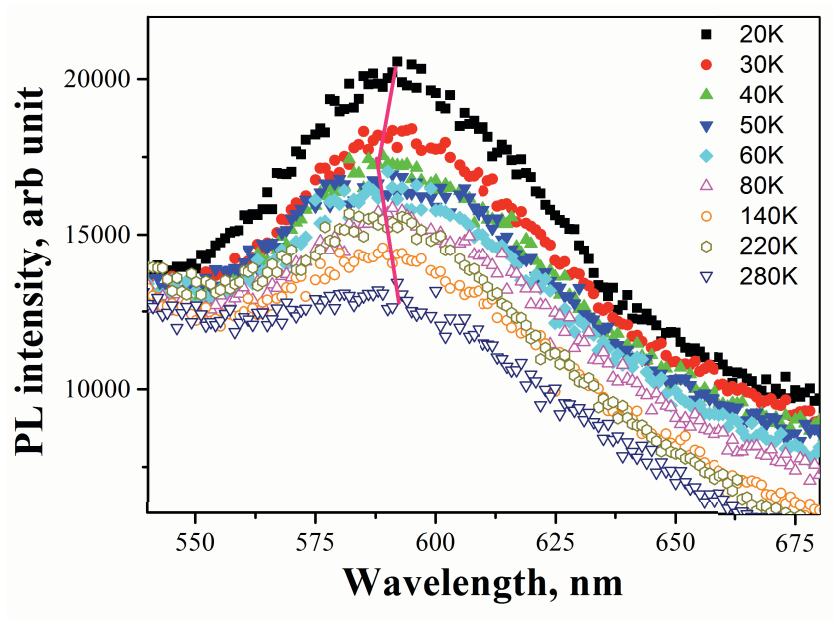

Figure. 5. Temperature dependent photoluminescence spectra of the CdSe nanoparticles measured from $20 \mathrm{~K}$ to $280 \mathrm{~K}$ under $405 \mathrm{~nm}$ laser excitation. 


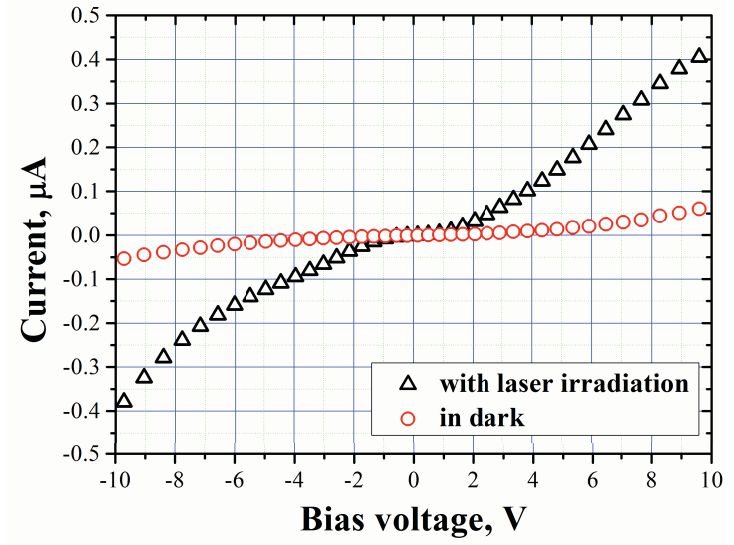

Figure. 6. I-V curves of the CdSe nanoparticle arrays measured with and without light illumination.

\section{Conclusions}

We used a high vacuum cluster beam deposition system to prepare CdSe NPs. The NPs were generated in gas phase with a magnetron plasma gas aggregation cluster source. The formation of CdSe NPs in gas phase is based on homogeneous nucleation with the present of inert buffer gas and subsequent condensation and growth. The gas-phase process have the advantages such as high purity, clean particle surface and interface, good process and product control, as well as mass production. Coagulation-free CdSe nanocrystal arrays with a fairly uniform spatial distribution were obtained. The NPs have a good dispersity with a mean diameter of about $4.8 \mathrm{~nm}$. The band-gap energy of the CdSe NPs was about $2.8 \mathrm{eV}$, with a significant bule shift relative to bulk CdSe, which was induced by the quantum confinement effect. A strong PL band corresponding to the near-band-edge transition of the CdSe NPs was observed. The CdSe NP films show a significant photoconductance induced by $405 \mathrm{~nm}$ laser irradiation. With an applied bias voltage of $10 \mathrm{~V}$, the photo-induced current can be as high as $0.4 \mu \mathrm{A}$ under $0.01 \mu \mathrm{W} / \mathrm{mm}^{2}$ light intensity. Our approach offers an alternative method for CdSe NP synthesis as compared to the existing methods.

\section{Acknowledgments}

This work was supported by the Jiangsu Province Innovation Fund for Technology Based Firms (BC2013118).

\section{References}

1. M.C.Schlamp, Peng, X.G., A.P.Alivisatos, Improved efficiencies in light emitting diodes made with $\mathrm{CdSe}(\mathrm{CdS})$ core/shell type nanocrystals and a semiconducting polymer, J. Appl. Phys. 82(1997) 5837-5842.

2. J. M. Caruge, J. E. Halpert, V. Wood, V. Bulovic, M. G. Bawendi, Light emitting diodes made with epitaxially grown $\mathrm{CdSe} / \mathrm{CdS}$ core/shell nanocrystals, Nat. Photonics 2 (2008) 247-250.

3. X. Michalet, F. Pinaud, T.D.Lacoste, M. Dahan, M. P. Bruchez, A. P. Alivisatos, S. Weiss, Properties of
Fluorescent Semiconductor Nanocrystals and their Application to Biological Labeling. Single Mol. 2 (2001) 261-276.

4. W. C. W. Chan, D. J. Maxwell, R. E. Bailey, M. Y. Han, S. M. Nie, Luminescent quantum dots for multiplexed biological detection and imaging. Curr. Opin. Biotechnol. 13(2002)40-46.

5. M. Buchez Jr, M. Moronne, P. Gin, S. Weiss, A. P. Alivisatos, Semiconductor Nanocrystals as Fluorescent Biological Labels, Science 281 (1998) 2013.

6. V.L. Colvin, M.C. Schlamp, A.P. Alivisatos, Lightemitting diodes made from cadmium selenide nanocrystals and a semiconducting polymer, Nature 370(1994)354- 357.

7. V. I. Klimov, A. A. Mikhailovsky, S.Xu, A. Malko, H.-J. Eisler, M. G.Bawendi, Optical Gain and Stimulated Emission in Nanocrystal Quantum Dots, Science 290(2000) 314-317.

8. J. H. Bang, P. V. Kamat, Quantum dot sensitized solar cells. A tale of two semiconductor nanocrystals: CdSe and CdTe, ACS Nano 3(2009)1467-1476.

9. A. Kongkanand, K. Tvrdy, K. Takechi, M. Kuno, P. V. Kamat, Quantum dot solar cells. Tuning photoresponse through size and shape control of CdSe-TiO2 architecture, J. Am. Chem. Soc. 130(2008) 4007-4015.

10. N. G. Semaltianos, S. Logothetidis, W. Perrie, S. Romani, M. Sharp, P. French, G. Dearden, K. G. Watkins, CdSe nanoparticles synthesized by laser ablation, Europhys. Lett. 84 (2008) 47001.

11. Y. T. Didenko, K. S. Suslick, Chemical Aerosol Flow Synthesis of Semiconductor Nanoparticles, J. Am. Chem. Soc. 127(2005)12196-12197.

12. M. Han, C. H. Xu, D. Zhu, L. Yang, J. L. Zhang, Y. P. Chen, K. Ding, F. Q. Song, G. H. Wang, Controllable Synthesis of Two-dimensional Metal Nanoparticle Arrays with Oriented Size and Number Density Gradients, Adv. Mater. 19 (2007) 2979-2983.

13. H. Haberland, M. Mall, M. Moseler, Y. Qiang, T. Reiners, and Y. Thurner, J. Vac. Sci. Technol. A 12(1994) 2925.

14. A. M. Kelley,Q.Q.Dai,Z. J. Jiang,J. A. Baker,D. F. Kelley, Resonance Raman spectra of wurtzite and zincblende CdSe nanocrystals, Chem. Phys. 422(2013) 272-276.

15. V. M. Dzhagan, M. Y. Valakh, A. E. Reavskaya, A. L. Stroyuk, S. Y. Kuchmiy, D. R. T. Zahn, Resonant Raman scattering study of CdSe nanocrystals passivated with $\mathrm{CdS}$ and $\mathrm{ZnS}$ Nanotechnology, 18(2007)285701.

16. V.M. Dzhagan, M.Y. Valakh, A.E. Reavskaya, A.L. Stroyuk, S.Y. Kuchmiy, D.R.T. Zahn, Size effects on Raman spectra of small CdSe nanoparticles in polymer films, Nanotechnology 19(2008)305707.

17. Z. Ma, K. Pierz, P. Hinze, Abnormal temperature behavior of photoluminescence from self-assembled InAs/AlAs quantum dots, Appl. Phys. Lett., 79(2001), $2564-2566$. 